\title{
Precise Species Identification by Whole-Genome Sequencing of Enterobacter Bloodstream Infection, China
}

\author{
Wenjing Wu, ${ }^{1}$ Li Wei, ${ }^{1}$ Yu Feng, Yi Xie, Zhiyong Zong
}

\begin{abstract}
The clinical importance of Enterobacter spp. remains unclear because phenotype-based Enterobacter species identification is unreliable. We performed a genomic study on 48 cases of Enterobacter-caused bloodstream infection by using in silico DNA-DNA hybridization to identify precise species. Strains belonged to 12 species; Enterobacter xiangfangensis $(\mathrm{n}=21)$ and an unnamed species (taxon $1, \mathrm{n}=8$ ) were dominant. Most $(63.5 \%)$ Enterobacter strains $(n=349)$ with genomes in GenBank from human blood are E. xiangfangensis; taxon 1 $(19.8 \%)$ was next most common. E. xiangfangensis and taxon 1 were associated with increased deaths $(20.7 \%$ vs. $15.8 \%$ ), lengthier hospitalizations (median $31 \mathrm{~d}$ vs. $19.5 \mathrm{~d}$ ), and higher resistance to aztreonam, cefepime, ceftriaxone, piperacillin-tazobactam, and tobramycin. Strains belonged to 37 sequence types (STs); ST171 ( $E$. xiangfangensis) was most common $(n=6)$. Four ST171 strains belonged to a defined clone. Precise species identification has greater implications for epidemiology and infection control than treatment.
\end{abstract}

Fnterobacter spp. belongs to the family EnterobacteEriaceae and is a common pathogen in a variety of infections, such as bloodstream and intraabdominal infections, most of which are healthcare associated (1). Enterobacter spp. is the third most common human pathogen, after Escherichia coli and Klebsiella pneumoniae, and is therefore of clinical importance (1). Enterobacter consists of several closely related species (1) that cannot typically be identified precisely by common phenotypic tests. The taxonomy of Enterobacter is complicated by the reassignment to other genera of some species that formerly belonged to the Enterobacter genus. For example, E. aerogenes has been moved to genus Klebsiella

Author affiliations: West China Hospital, Sichuan University, Chengdu, China (W. Wu, L. Wei, Y. Feng, Y. Zie, Z. Zong); State Key Laboratory of Biotherapy, Chengdu (W. Wu, Y. Feng, Z. Zong)

DOI: https://doi.org/10.3201/eid2701.190154
(2), E. agglomerans to genus Pantoea (3), and E. sakazakii to genus Cronobacter (4). Currently, 14 Enterobacter spp. with validly published names exist, and 3 additional Enterobacter spp. have tentative species designations awaiting validation under the rules of the International Code of Nomenclature of Bacteria (Bacteriological Code) (Appendix 1 Table 1, https://wwwnc.cdc.gov/EID/ article/27/1/19-0154-App1.pdf).

Several Enterobacter spp., such as E. asburiae, E. cloacae, and E. hormaechei, cause infections in humans (1). Enterobacter strains extracted from clinical samples are usually reported as E. cloacae, and sometimes E. asburiae, E. hormaechei, or E. kobei, by automated microbial identification systems such as Vitek II (bioMérieux, https://www.biomerieux.com). However, such phenotype-based tests are unreliable for species identification of Enterobacter and can result in misidentification (1). For instance, all Enterobacter spp. have a positive reaction for $\beta$-galactosidase, arginine dihydrolase, citrate utilization, sucrose, amygdalin, arabinose, and D-glucose but are negative for lysine decarboxylase, $\mathrm{H}_{2} \mathrm{~S}$ production, urease activity, indole production, deaminase, and gelatinase (5-7). Differentiating Enterobacter spp. by biochemical reactions commonly used in clinical microbiology laboratories is therefore difficult. The differences in clinical importance of each Enterobacter species remain largely unknown because they are regularly misidentified in clinical microbiology laboratories.

Because of the substantial reduction in cost of whole-genome sequencing for bacterial strains, we are entering the era of genomic microbiology (8). Newly created methods can determine the overall nucleotide identities between genome sequences and therefore enable more precise species identification (9). Calculation of average nucleotide identity (ANI) between genomes is widely used for species

${ }^{1}$ These authors contributed equally to this article. 
identification. It has been proposed that ANI $>96 \%$ would guarantee species assignation, whereas ANI of $<93 \%$ can be considered species differentiation (10). However, ANI values in the range of 93\%-96\% represent a vague zone in which the boundary of a species might fall (10). DNA-DNA hybridization (DDH) remains the standard for species identification, with a $\geq 70 \%$ cutoff recommended to define a species. However, DDH is cumbersome, prone to fluctuation, and requires the availability of type strains. To overcome the shortcomings of $\mathrm{DDH}$, in silico DDH (isDDH) mimics DDH by comparing genome sequences and can be a reliable and convenient tool for species assignation. To provide insight into the potential clinical importance of different $E n$ terobacter spp., we performed a genomic study using isDDH to identify bloodstream infection (BSI)-causing Enterobacter strains to the species level.

\section{Materials and Methods}

\section{Strain and Susceptibility Tests}

We collected nonduplicate Enterobacter strains recovered from blood cultures during January 2016June 2018 at West China Hospital of Sichuan University (Appendix 2 Table 1, https://wwwnc.cdc. gov/EID/article/27/1/19-0154-App2.xlsx). West China Hospital is a 5,000-bed major referral hospital in western China. Initial species identification and in vitro susceptibility testing were performed by using Vitek II. We determined MICs of colistin by using the broth microdilution method of the Clinical and Laboratory Standards Institute (CLSI) and interpreted susceptibility following CLSI guidelines (11). For colistin and tigecycline, no CLSI breakpoints are available, so we used breakpoint standards defined by the European Committee on Antimicrobial Susceptibility Testing (https://www.eucast.org). Multidrug resistance was defined based on the criteria for Enterobacteriaceae (12).

\section{Patient Data}

West China Hospital has a comprehensive hospital information system, which allowed us to retrieve patient data including age, sex, length of hospitalization, and clinical outcomes (death or discharge) from electronic medical records. One patient (with strain 090040) had an unusually long hospital stay (578 d) because of a medical dispute and was removed from our analysis of length of stay. According to social customs in China, dying at home is preferred over the hospital; it is likely many patients chose to stop treatment and return home if treatment was not working and patients felt death was imminent. We categorized patients who chose to be discharged but were likely to die in the hospital (judged by the consensus of 2 physicians reviewing blind data) as patients with predicted death. BSI, the type of BSI (primary or secondary to infection of other sites), central line-associated BSI (CLABSI), and healthcare-associated infection were determined by using criteria established by the Centers for Disease Control and Prevention's National Healthcare Safety Network $(13,14)$. We conducted the study in accordance with the amended Declaration of Helsinki. The Ethics Committee of West China Hospital approved the study and waived informed consent.

\section{Short-Read Genome Sequencing, Analysis, and Precise Species Identification}

All strains underwent whole-genome sequencing by using the HiSeq X10 platform (Illumina, https://www.illumina.com). Genomic DNA was prepared by using the QIAamp DNA mini kit (QIAGEN, https://www.qiagen.com). We used Unicycler version $0.4 .3(15)$, in the conservative mode for increased accuracy, to perform a de novo hybrid assembly. Precise species identification was established by determining the pairwise isDDH between the genome sequence of the query strain and those of type strains of Enterobacter spp., including the validly published species and the species awaiting validation (Appendix 1 Table 1). This process was performed by using the Genome-to-Genome Distance Calculator, formula 2 (16). A $\geq 70 \%$ cutoff was applied to define a species. In addition, we determined the pairwise ANI of the genome sequence of the query strain and those of type strains of Enterobacter spp. (Appendix 1 Table 1) by using JSpecies software (https://imedea.uib-csic.es/ jspecies) with a $>96 \%$ ANI cutoff to define a species (10). Sequence types (STs) were determined by using the genomic sequences to query the multilocus sequence typing database of E. cloacae (https:/ / pubmlst.org/ecloacae). Antimicrobial resistance genes were identified from genome sequences by using the ABRicate program (https://github.com/ tseemann/abricate) to query the ResFinder database (https:/ / genomicepidemiology.org).

The genome sequences of all Enterobacter strains recovered from human blood $(\mathrm{n}=349$, Appendix 2 Table 2) were retrieved from GenBank (accessed 2018 Nov 1). These Enterobacter genomes were subjected to precise species identification by using the Genometo-Genome Distance Calculator as described. 


\section{Clonal Relatedness on the Basis of Single-Nucleotide Polymorphisms}

We performed single-nucleotide polymorphism (SNP) calling for genome sequences to untangle the clonal relatedness of ST171 strains and to investigate whether the ST171 strains in this study are clonally related to strains recovered elsewhere. All genome sequences of ST171 Enterobacter strains ( $\mathrm{n}=102)$, regardless of types of the host source (human, nonhuman, or unknown) and source (blood, nonblood, or unknown), were retrieved from GenBank. We used the complete chromosome sequence of ST171 strain 34798 (GenBank accession no. CP012165), which was recovered from a bile sample in the United States in 2011 , as our reference for mapping. Genome sequences of the strains were mapped against the reference genome by using Snippy version 4.3.6 (https:/ / github. $\mathrm{com} /$ tseemann/snippy) at default settings. The resulting core SNPs $(n=1,918)$ were concatenated and used to infer a phylogenomic tree by using RAxML version 8.2.12 (17) under the general time-reversible plus gamma model with a 1,000-bootstrap test.

\section{Long-Read Genome Sequencing and Plasmid Analysis for ST171 Strains}

We determined plasmid replicons of all ST171 strains in this study by using PlasmidFinder version 2.0 (https:/ / cge.cbs.dtu.dk/services/PlasmidFinder). The first $b l a_{\mathrm{NDM}-5}-$ harboring ST171 strain (090011) in this study and the only $b l a_{\mathrm{NDM}-1}-$ harboring ST171 strain (045001) were selected for whole-genome sequencing by using the long-read MinION sequencer (Nanopore, https://nanoporetech.com) to obtain complete chromosomal and plasmid sequences. De novo hybrid assembly of short Illumina reads and long MinION reads was performed by using Unicycler version 0.4.3 (15) in conservative mode for increased accuracy. Complete circular contigs were then corrected by using Pilon version 1.22 (18) with Illumina reads for several rounds until no further improvements were reported. Short reads of the remaining four $b l a_{\mathrm{NDM}-5}$-harboring ST171 strains (090022, 090023, 090055, and 090059) were mapped against the $b l a_{\mathrm{NDM}-5}$-carrying plasmid (designated pNDM5_090011) of strain 090011 by using BWA version 0.7.17 (H. Ling, unpub. data, https:// arxiv.org/abs/1303.3997) at default settings.

\section{Statistical Analysis}

Continuous variables were presented as median and interquartile range and were compared by using rank-sum test. We used the Pearson $\chi^{2}$ test, Yates correction for continuity, or Fisher exact test to compare disparities between different groups for categorical variables. Pearson $\chi^{2}$ test was used when sample size (n) was $\geq 40$ and theoretical frequency $(\mathrm{T}) \geq 5$, Yates correction for continuity when $\mathrm{n} \geq 40$ and $1 \leq \mathrm{T}<5$, and Fisher exact test when $\mathrm{n}<40$ or $\mathrm{T}<1$. We used SPSS Statistics 21.0 (IBM Inc., https:/ / www.ibm.com) to perform statistical analyses. All $p$ values were 2-tailed, and $p<0.05$ was considered statistically significant. We used PASS version 11.0 (NCSS, https:/ / www.ncss.com) to calculate statistical power after using the Wilcoxon test to conduct nonparametric adjustment.

Draft genome sequences of the strains in this study were deposited into GenBank (accession numbers in Appendix 2 Table 1). The complete genome sequence of strain 090011 was deposited into GenBank under accession nos. CP036310-2. and the sequence for strain 045001 was deposited under accession nos. CP043382-5.

\section{Results}

\section{Precise Species Identification of Enterobacter Strains}

A total of 48 nonduplicated Enterobacter strains were recovered from blood during our 2.5-year study period and were collected for study (Table 1; Appendix 2 Table 1). Whole-genome sequencing results, isDDH, and ANI values of these strains are summarized in Appendix 2 Table 1. The 48 strains were identified as E. cloacae (n $=42)$, E. asburiae $(\mathrm{n}=3)$, and E. kobei $(\mathrm{n}=3)$ by Vitek II. However, precise species identification on the basis of isDDH revealed that the most common species was actually E. xiangfangensis $(\mathrm{n}=21)$ (Table 2); the next most common was an Enterobacter sp. $(\mathrm{n}=8)$ that has no assigned species name but was previously known as Enterobacter cluster III, as defined by Hoffman et al. (6). This species is most closely related to E. xiangfangensis with a $66.6 \%$ isDDH value and is temporarily assigned taxon 1 here (Appendix 2 Table 2). The remaining strains were assigned to 10 Enterobacter species: E. bugandensis $(\mathrm{n}=4)$, E. cloacae $(\mathrm{n}=3)$, E. asburiae $(\mathrm{n}=2)$, E. hormaechei $(\mathrm{n}=2)$, E. huaxiensis $(\mathrm{n}=2)$, E. roggenkampii $(\mathrm{n}=2)$, E. chuandaensis $(\mathrm{n}=1)$, E. ludwigii $(\mathrm{n}=1)$, E. sichuanensis $(\mathrm{n}=1)$, and an unnamed Enterobacter sp. $(\mathrm{n}=1)$ (Table 2). The unnamed species is most closely related to $E$. roggenkampii with a $65.4 \%$ isDDH value (Appendix 1 Table 2 ) and is temporarily assigned taxon 2 here.

Of 349 Enterobacter strains in GenBank that were recovered from human blood, most $(221,63.3 \%)$ are also E. xiangfangensis; taxon 1 is the second most common species with 69 strains (19.8\%) (Table 2). The remaining 59 strains (16.9\%) belong to 14 species: E. asburiae $(\mathrm{n}=14[4.0 \%])$, E. kobei $(\mathrm{n}=10$ [2.9\%]), E. bugandensis $(\mathrm{n}=7[2.0 \%])$, E. roggenkampii $(\mathrm{n}=7[2.0 \%])$, 
Table 1. Enterobacter strains in genomic study of Enterobacter bloodstream infection, China*

\begin{tabular}{|c|c|c|c|c|c|c|c|c|c|c|c|}
\hline \multirow[b]{2}{*}{ Strain } & \multirow[b]{2}{*}{ Date } & \multirow[b]{2}{*}{ Species, by isDDH } & \multirow[b]{2}{*}{ ST† } & \multirow[b]{2}{*}{ Carbapenemase } & \multicolumn{4}{|c|}{ BSI types } & \multicolumn{2}{|c|}{ Hospitalization, d } & \multirow[b]{2}{*}{ Death } \\
\hline & & & & & $\mathrm{P}$ & $\mathrm{S}$ & CLA & $\mathrm{HA}$ & Before BSI & After BSI & \\
\hline 090034 & 201706 & Enterobacter asburiae & N12 & & + & & & & 0 & 27 & \\
\hline 090058 & 201805 & E. asburiae & 879 & & + & & + & + & 22 & 18 & \\
\hline 090031 & 201711 & E. bugandensis & N10 & & + & & & & 1 & 0 & + \\
\hline 090283 & 201610 & E. bugandensis & N16 & & & + & & + & 7 & 6 & \\
\hline 090210 & 201607 & E. bugandensis & 499 & & & + & & & 1 & 16 & \\
\hline 090029 & 201709 & E. bugandensis & 718 & NDM-5 & + & & & + & 71 & 16 & + \\
\hline 090028 & 201708 & E. chuandaensis & N9 & & & + & & & 1 & 11 & \\
\hline 090005 & 201706 & E. cloacae & 1 & NDM-1 & + & & + & + & 29 & 11 & \\
\hline 090016 & 201703 & E. cloacae & 519 & & & + & & + & 17 & 24 & \\
\hline 090014 & 201712 & E. cloacae & 922 & & & + & & + & 0 & 10 & \\
\hline 090027 & 201704 & E. hormaechei & 528 & & & + & & + & 24 & 38 & \\
\hline 090003 & 201705 & E. hormaechei & 696 & & + & & & + & 0 & 15 & \\
\hline 045002 & 201609 & E. huaxiensis & N1 & & + & & & & 0 & 7 & \\
\hline 090008 & 201709 & E. huaxiensis & N1 & & + & & & + & 3 & 23 & \\
\hline 090017 & 201702 & E. ludwigii & 12 & & & + & & + & 6 & 8 & \\
\hline 045158 & 201607 & E. roggenkampii & N2 & & + & & & + & 3 & 7 & \\
\hline 090037 & 201608 & E. roggenkampii & 984 & & + & & + & + & 14 & 8 & \\
\hline 090032 & 201712 & E. sichuanensis & N11 & & + & & + & + & 6 & 16 & + \\
\hline 090004 & 201706 & E. xiangfangensis & N3 & & + & & & + & 6 & 13 & \\
\hline 090006 & 201707 & E. xiangfangensis & N4 & & + & & & + & 12 & 8 & \\
\hline 090007 & 201707 & E. xiangfangensis & N5 & & + & & & + & 24 & 19 & \\
\hline 090012 & 201711 & E. xiangfangensis & N6 & & & + & & & 0 & 3 & \\
\hline 090018 & 201705 & E. xiangfangensis & N7 & & + & & + & + & 12 & 14 & + \\
\hline 090020 & 201712 & E. xiangfangensis & N8 & & + & & + & + & 4 & 60 & \\
\hline 090057 & 201804 & E. xiangfangensis & N15 & NDM-1 & + & & + & + & 36 & 43 & \\
\hline 090015 & 201712 & E. xiangfangensis & 50 & & + & & & & 0 & 2 & \\
\hline 090026 & 201704 & E. xiangfangensis & 50 & & + & & & + & 11 & 52 & \\
\hline 090035 & 201712 & E. xiangfangensis & 127 & & + & & & + & 13 & 20 & \\
\hline 045001 & 201801 & E. xiangfangensis & 171 & NDM-1 & + & & + & + & 8 & 33 & \\
\hline 090011 & 201710 & E. xiangfangensis & 171 & NDM-5 & + & & & + & 7 & 14 & + \\
\hline 090022 & 201802 & E. xiangfangensis & 171 & NDM-5 & + & & & + & 40 & 20 & \\
\hline 090023 & 201802 & E. xiangfangensis & 171 & NDM-5 & + & & + & + & 26 & 19 & \\
\hline 090055 & 201806 & E. xiangfangensis & 171 & NDM-5 & & + & & + & 14 & 14 & \\
\hline 090059 & 201805 & E. xiangfangensis & 171 & NDM-5 & + & & + & + & 17 & 31 & \\
\hline 090043 & 201612 & E. xiangfangensis & 337 & & & + & & + & 11 & 4 & + \\
\hline 090013 & 201711 & E. xiangfangensis & 418 & NDM-5 & + & & + & + & 28 & 10 & + \\
\hline 090038 & 201609 & E. xiangfangensis & 418 & & + & & & + & 3 & 33 & \\
\hline 090060 & 201806 & E. xiangfangensis & 550 & & + & & + & + & 20 & 8 & + \\
\hline 090042 & 201611 & E. xiangfangensis & 828 & & + & & & + & 5 & 3 & \\
\hline 090036 & 201605 & Taxon 1 & N13 & & + & & + & + & 13 & 22 & \\
\hline 090019 & 201706 & Taxon 1 & 78 & & + & & & + & 16 & 62 & \\
\hline 090030 & 201709 & Taxon 1 & 78 & & + & & + & + & 0 & 15 & \\
\hline 090039 & 201610 & Taxon 1 & 78 & & & + & & + & 12 & 8 & + \\
\hline 090021 & 201801 & Taxon 1 & 97 & & + & & + & + & 5 & 28 & \\
\hline 090009 & 201709 & Taxon 1 & 104 & & & + & & + & 5 & 66 & \\
\hline 090033 & 201704 & Taxon 1 & 316 & & + & & & + & 10 & 3 & \\
\hline 090056 & 201803 & Taxon 1 & 568 & & + & & & + & 11 & 7 & \\
\hline 090040 & 201611 & Taxon 2 & N14 & & + & & + & + & 208 & 370 & \\
\hline
\end{tabular}

*BSI, bloodstream infection; CLA, central line-associated; HA, healthcare associated; isDDH, in silico DNA-DNA hybridization; P, primary; S, secondary; ST, sequence type.

†There are 16 new sequence types, which are temporarily assigned N1-N16 (Appendix 1 Table 3, https://wwwnc.cdc.gov/EID/article/27/1/19-0154App1.pdf).

E. ludwigii $(\mathrm{n}=6[1.7 \%])$, E. cloacae $(\mathrm{n}=4[1.1 \%])$, taxon $1(\mathrm{n}=2)$, E. chengduensis $(\mathrm{n}=1)$, E. mori $(\mathrm{n}=1)$, E. sichuanensis ( $\mathrm{n}=1)$, and 4 species without assigned species names ( $n=1$ or 2 for each species, 6 in total; Table 2). The 4 unnamed species were assigned taxon 3-6 (Table 2); the closest species of taxon 3-6 are listed in Appendix 1 Table 2 and are also shown in a phylogenomic tree in Appendix 1 Figure 1. E. xiangfangensis and taxon 1 are closely related as shown by their phylogenetic position in the phylogenomic tree of Enterobacter spp. and by their common $66.6 \%$ isDDH value (close to the $70 \%$ cutoff to define a species). We therefore combined the 2 species in the following analysis.

\section{BSI Types and Characteristics}

Most of the 48 BSI cases ( $\mathrm{n}=36,75 \%$ ) were primary BSIs, including 16 cases of CLABSI. BSIs in the remaining 12 cases were secondary; original sources were intraabdominal infection $(n=5)$, cholangitis ( $n$ $=3)$, urinary tract infection $(n=2)$, wound infection 
$(\mathrm{n}=1)$, and gastrointestinal tract infection $(\mathrm{n}=1)$. Most ( $\mathrm{n}=41,85.4 \%$ ) BSIs caused by Enterobacter spp. were healthcare-associated infections. E. xiangfangensis and taxon 1 were more likely to cause primary BSI $(82.8 \%$ vs. $63.2 \%)$, CLABSI $(27.9 \%$ vs. $17.2 \%)$, and healthcare-associated BSI (93.1\% vs. $73.7 \%)$ than were other Enterobacter spp. However, the differences were not statistically significant (Table 3 ).

Two patients who had Enterobacter BSIs (1 E. xiangfangensis and 1 taxon 1) died in the hospital. In addition, 7 patients with Enterobacter BSIs (4 E. xiangfangensis, 2 E. bugandensis, and 1 E. sichuanensis) did not respond to treatment and were discharged in critical condition. These 7 case-patients were categorized as patients in whom death was predicted. The death rate for Enterobacter BSI was 18.8\% (9/48); the death rate $(20.7 \%$ [6/29]) of BSI caused by E. xiangfangensis or taxon 1 was not statistically different $(15.8 \%$ [3/19] $\mathrm{p}>0.05$ ) (Table 3) from that of BSI caused by other Enterobacter spp. Of note, 2 of the 4 patients with $E$. bugandensis-caused BSI had poor outcomes (predicted death) (Table 1). BSIs caused by E. xiangfangensis or taxon 1 were more common in younger patients and resulted in lengthier overall hospitalizations (median 33 vs. $19.5 \mathrm{~d} ; \mathrm{p}>0.05$ ) (Table 3) than BSIs caused by other Enterobacter spp. This difference was largely because of the prolonged length of stay (median $11 \mathrm{~d}$ vs. $4.5 \mathrm{~d} ; \mathrm{p}>0.05$ ) before the episode of BSI.

\section{Antimicrobial Susceptibility and Antimicrobial Resistance Genes}

The antimicrobial susceptibility and antimicrobial resistance gene repertoire of the 48 Enterobacter strains are shown in Appendix 2 Table 1. E. xiangfangensis and taxon 1 had substantially higher rates of resistance to aztreonam ( $48.3 \%$ vs. $10.5 \%)$, cefepime $(41.4 \%$ vs. $10.5 \%)$, ceftriaxone (58.6\% vs. $15.8 \%)$, piperacillin/ tazobactam (41.4\% vs. $10.5 \%)$, and tobramycin $(44.8 \%$
Table 2. Proportion of Enterobacter species recovered from blood in genomic study of Enterobacter bloodstream infection, China

\begin{tabular}{lcc}
\hline & \multicolumn{2}{c}{ No. (\%) } \\
\cline { 2 - 3 } Species* & $\begin{array}{c}\text { Strains from blood } \\
\text { in this study }\end{array}$ & $\begin{array}{c}\text { Strains from } \\
\text { blood in GenBank }\end{array}$ \\
\hline Enterobacter & $21(43.8)$ & $221(63.3)$ \\
xiangfangensis & & \\
Taxon 1 & $8(16.7)$ & $69(19.8)$ \\
E. bugandensis & $4(8.3)$ & $7(2.0)$ \\
E. cloacae & $3(6.3)$ & $4(1.1)$ \\
E. asburiae & $2(4.2)$ & $14(4.0)$ \\
E. roggenkampii & $2(4.2)$ & $7(2.0)$ \\
E. hormaechei & $2(4.2)$ & 0 \\
E. huaxiensis & $2(4.2)$ & 0 \\
E. ludwigii & $1(2.1)$ & $6(1.7)$ \\
E. sichuanensis & $1(2.1)$ & $1(0.3)$ \\
E. chuandaensis & $1(2.1)$ & 0 \\
Taxon 2 & $1(2.1)$ & $2(0.6)$ \\
E. kobei & 0 & $10(2.9)$ \\
Taxon 3 & 0 & $2(0.6)$ \\
Taxon 4 & 0 & $2(0.6)$ \\
Taxon 5 & 0 & $1(0.3)$ \\
Taxon 6 & 0 & $1(0.3)$ \\
E. chengduensis & 0 & $1(0.3)$ \\
E. mori & 0 & $1(0.3)$ \\
\hline Total & $48(100.0)$ & $349(100.0)$ \\
\hline${ }^{*}$ Taxons 1-6 represent 6 Enterobacter spp. without assigned names. Their \\
most closely related Enterobacter spp. are listed in Appendix 1 Table 2 \\
and shown in Appendix 1 Figure 1 \\
(https://wwwnc.cdc.gov/ElD/article/27/1/19-0154-App1.pdf). \\
\hline
\end{tabular}

vs. 5.3\%) (Table 4) and were substantially more likely to be multidrug resistant (55.2\% vs. 10.6\%) (Table 3). There were 10 carbapenem-resistant strains (8 E. xiangfangensis, 1 E. bugandensis, and 1 E. cloacae), all of which carried a $b l a_{\mathrm{NDM}}$ gene $\left(b l a_{\mathrm{NDM}-5}, \mathrm{n}=7 ; b l a_{\mathrm{NDM}-1,}, \mathrm{n}=3\right)(\mathrm{Ta}-$ ble 1; Appendix 2 Table 1), and they belonged to 5 STs $(\mathrm{ST} 171[\mathrm{n}=6]$, ST718 [n = 1], ST1 [n = 1], ST418 [n = 1], and a new ST $[n=1])$. No carbapenemase genes were identified in carbapenem-susceptible strains.

\section{Sequence Types and Clonal Relatedness}

The 48 Enterobacter strains belonged to 37 STs (Table 1; Appendix 1 Table 3) but only ST171 (E. xiangfangensis)

\begin{tabular}{|c|c|c|c|c|c|}
\hline Characteristic & $\begin{array}{l}\text { E. xiangfangensis and } \\
\text { taxon } 1, \mathrm{n}=29\end{array}$ & $\begin{array}{l}\text { Other species, } \mathrm{n} \\
\quad=19\end{array}$ & $\chi^{2}$ & $p$ value & Power† \\
\hline Age, y, median (IQR) & $15(8-32)$ & $52(16-71)$ & - & 0.958 & 0.085 \\
\hline Male sex & $19(65.5)$ & $14(73.7)$ & 0.356 & 0.551 & 0.084 \\
\hline MDR & $16(55.2)$ & $2(10.6)$ & 9.763 & 0.002 & - \\
\hline Primary BSI & $24(82.8)$ & $12(63.2)$ & 1.423 & 0.233 & 0.317 \\
\hline CLABSI & $11(27.9)$ & $5(17.2)$ & 0.697 & 0.404 & 0.115 \\
\hline HA BSI & $27(93.1)$ & $14(73.7)$ & 2.091 & 0.148 & 0.430 \\
\hline Deaths & $6(20.7)$ & $3(15.8)$ & 0.176 & 0.675 & 0.053 \\
\hline Total time hospitalized, d, median (IQR)‡ & $33(19-47)$ & $20(2-40)$ & - & 0.098 & 0.265 \\
\hline Time hospitalized before BSI onset, $d$, median (IQR) $\ddagger$ & $11(5-7)$ & $5(1-18)$ & - & 0.154 & 0.070 \\
\hline Time hospitalized after BSI onset, d, median (IQR) & $15(8-32)$ & $13(8-19)$ & - & 0.357 & 0.384 \\
\hline
\end{tabular}


and ST78 (taxon 1) contained $\geq 3$ strains ( 6 for ST171 and 3 for ST78). We performed analysis of clonal relatedness based on SNPs for ST78 and ST171 strains. In the 3 ST78 strains, there were 306 to 1,052 SNPs difference, suggesting no recent shared origins (Appendix 1 Table 4). The 6 ST171 strains were all resistant to carbapenems and carried $b l a_{\mathrm{NDM}-5}(\mathrm{n}=5)$ or $b l a_{\mathrm{NDM}-1}(\mathrm{n}=1)$. Among the 6 ST171 strains, 4 (all carrying $b l a_{\text {NDM-5 }}$ ) had 0-2 SNPs difference (Table 5) and were recovered from patients in the same ward (cardiac surgery). One patient infected with strain 090011 died but the remaining 3 patients recovered.

The remaining 2 ST171 strains, 045001 (carrying $\left.b l a_{\mathrm{NDM}-1}\right)$ and 090055 (carrying $b l a_{\mathrm{NDM}-5}$ ), were 81-82 SNPs different from the 4 previously mentioned strains and were 38 SNPs different from each other. These 2 strains were recovered from patients in 2 different wards (medical and respiratory intensive care units). The 6 ST171 strains isolated in our study formed a phylogenetic cluster with strain CCBH10892, which was isolated from a rectal swab sample in Brazil in 2012 (GenBank accession no. JSBO00000000), and strain EC_849, which was isolated from a sputum sample in South Africa in 2012 (GenBank accession no. LRIZ00000000) (Appendix 1 Figure 2). The cluster contained 98 to 107 SNPs difference (Table 5; Appendix 1 Figure 2). Of note, both CCBH10892 and EC_849 carried $b l a_{\mathrm{NDM}-1}$. By contrast, the 6 strains were $>300$ SNPs different from other ST171 strains with genome sequences deposited in GenBank (Appendix 2 Table 3).

\section{Plasmid Analysis of ST171 Strains}

The complete genome sequences of $b l a_{\mathrm{NDM}-5}$-harboring strain 090011 and the $b l a_{\mathrm{NDM}-1}$-harboring strain 045001 were obtained. Strain 090011 had a 4.64-Mb circular chromosome and 2 plasmids (a 102.5-kb plasmid containing IncFIA, IncFIB, and IncR replicons and a 46.1-kb plasmid containing an IncX3 replicon) (Appendix 1 Table 5). The $b l a_{\mathrm{NDM}-5}$ gene in strain 090011 was carried on the $46.1-\mathrm{kb}$ IncX3 plasmid, designated pNDM5_090011. The short reads of the remaining $4 b^{b l a_{N D M}-5}$-harboring strains were then mapped against pNDM5_0900117. The 4 strains had contigs showing 100\% coverage and 100\% identity with pNDM5_090011, suggesting a common plasmid in all 5 bla $a_{\mathrm{NDM}-5}$-harboring ST171 strains in our study. Strain 045001 had a $4.70-\mathrm{Mb}$ circular chromosome and 3 plasmids (an 85.7-kb IncFII plasmid, a 78.2-kb plasmid, and a $2.5-\mathrm{kb}$ plasmid) (Appendix 1 Table 5). The replicon type of the latter 2 plasmids could not be determined by the current replicon-typing scheme. The $b l a_{\mathrm{NDM}-1}$ gene in strain 045001was carried on the 85.7-kb IncFII plasmid.

\section{Discussion}

Although genome sequences deposited in GenBank might be biased in sampling, they can provide complementary information on the species distribution of Enterobacter in cases of BSI. Examination of our set of strains and those available in GenBank demonstrates that a variety of Enterobacter spp. can cause BSI, but most BSI-causing Enterobacter strains belong to either E. xiangfangensis or, less commonly, taxon 1. E. xiangfangensis and taxon 1 are closely related, with a $66.6 \%$ isDDH value (near the $70 \%$ cutoff to define a species). Why the 2 species account for most Enterobacter BSIs, however, remains unknown. Although the colonization of the human gastrointestinal tract by Enterobacter has not been investigated to the level of precise species identification, E. xiangfangensis and taxon 1 could be the most common Enterobacter species colonizing there, which warrants further study.

Table 4. Antimicrobial resistance rates in E. xiangfangensis plus taxon 1 and other Enterobacter spp. in genomic study of Enterobacter bloodstream infection, China*

\begin{tabular}{|c|c|c|c|c|c|}
\hline \multirow[b]{2}{*}{ Antimicrobial agent } & \multicolumn{2}{|c|}{ No. (\%) } & \multirow[b]{2}{*}{$\chi^{2}$} & \multirow[b]{2}{*}{$p$ value } & \multirow[b]{2}{*}{ Powert } \\
\hline & $\begin{array}{l}\text { E. xiangfangensis } \\
+ \text { taxon } 1, n=29\end{array}$ & Other species, $n=19$ & & & \\
\hline Amikacin & $2(6.9)$ & 0 & - & 0.512 & ND \\
\hline Gentamicin & $9(31.0)$ & $1(5.3)$ & 3.192 & 0.074 & 0.600 \\
\hline Tobramycin & $13(44.8)$ & $1(5.3)$ & 8.698 & 0.003 & - \\
\hline Aztreonam & $14(48.3)$ & $2(10.5)$ & 7.361 & 0.007 & 0.829 \\
\hline Cefepime & $12(41.4)$ & $2(10.5)$ & 5.289 & 0.021 & - \\
\hline Ceftriaxone & $17(58.6)$ & $3(15.8)$ & 8.664 & 0.003 & - \\
\hline Imipenem & $8(27.6)$ & $2(10.5)$ & 1.123 & 0.289 & 0.258 \\
\hline Piperacillin/tazobactam & $12(41.4)$ & $2(10.5)$ & 5.289 & 0.021 & - \\
\hline Ciprofloxacin & $11(37.9)$ & 0 & 7.326 & 0.007 & ND \\
\hline Levofloxacin & $11(37.9)$ & 0 & 7.326 & 0.007 & ND \\
\hline Colistin & $10(34.5)$ & $6(31.6)$ & 0.044 & 0.835 & 0.052 \\
\hline Tigecycline & $5(17.2)$ & 0 & 2.043 & 0.153 & ND \\
\hline Trimethoprim/sulfamethoxazole & $12(41.4)$ & 0 & 8.392 & 0.004 & ND \\
\hline
\end{tabular}

*Bold indicates significance. ND, not determined; - , not calculated.

†Statistical power was calculated for parameters without statistical significance $(p>0.05)$ but could not be calculated for any parameters being 0 . 
Enterobacter Bloodstream Infection, China

Table 5. Single-nucleotide polymorphisms between the 6 ST171 strains in genomic study of Enterobacter bloodstream infection, China, and strain EC 849 from South Africa and strain CCBH10892 from Brazil*

\begin{tabular}{lcccccccc}
\hline Strain & 090011 & 090022 & 090023 & 090059 & 045001 & 090055 & EC_849 & CCBH10892 \\
\hline 090011 & - & 1 & -1 & 1 & 1 & 81 & 81 & 106 \\
090022 & 1 & 1 & - & 1 & 82 & 82 & 107 & 102 \\
090023 & 1 & 2 & 1 & - & 82 & 81 & 106 & 102 \\
090059 & 81 & 82 & 81 & 82 & - & 38 & 103 & 103 \\
045001 & 81 & 82 & 81 & 82 & 38 & - & 103 & 99 \\
090055 & 106 & 107 & 106 & 107 & 103 & 103 & - & 98 \\
EC_849 & 102 & 103 & 102 & 103 & 99 & 99 & 98 & - \\
CCBH10892 & & &
\end{tabular}

Alternatively, E. xiangfangensis and taxon 1 could be more pathogenic than other Enterobacter spp., which also requires further study.

BSIs caused by E. xiangfangensis and taxon 1 were more likely to occur in younger patients and result in longer overall hospital stays, although the differences in length of hospitalization between the 2 groups were not statistically significant $(p>0.05)$. This finding might be because of the relatively small sample size (power <0.8) (Table 3). Resistance rates to certain antimicrobial agents, including aztreonam, cefepime, ceftriaxone, piperacillin/tazobactam, and tobramycin and prevalence of multidrug resistance in E. xiangfangensis and taxon 1 were substantially higher than in other Enterobacter spp. This difference suggests that the identification of Enterobacter strains to precise species level also has implications in options of antimicrobial treatment. Although BSI caused by E. xiangfangensis and taxon 1 was not associated with higher death rates, 2 of the 4 patients with E. bugandensis had poor outcomes (predicted death). E. bugandensis has been reported to be a highly pathogenic species associated with life-threatening BSIs and sepsis (19). The virulence of this species warrants further study.

As previously noted, $4 b_{\mathrm{NDM}-5}$-harboring ST171 strains had 0-2 SNPs difference (Table 5) and were taken from patients in the same ward (cardiac surgery). The first patient with BSI caused by a strain belonging to the clone (090011) had Enterobacter BSI before being transferred to West China Hospital from another facility. Although the particular Enterobacter strain from the first hospital was not available for analysis, it is very likely that 090011 was introduced to West China Hospital by transfer of this patient. The next 3 cases were acquired in West China Hospital, highlighting both interhospital and intrahospital transmission of a common strain. BSI in 2 of the 3 cases was CLABSI. These findings suggest that the 4 strains belong to a common clone that caused a cluster of BSI cases. In the hospital ward, central lines were commonly used for drawing blood but were not properly decontaminated after each use; in addition, healthcare workers were observed by anonymous interns to have low compliance $(24.8 \%)$ with hand hygiene standards established by the World Health Organization. There have been no further Enterobacter BSIs after restricting access to central lines and promoting hand hygiene among healthcare workers, which resulted in the compliance rate increasing to $45.5 \%$.

The remaining 2 ST171 strains, 045001 (harboring $b l a_{\mathrm{NDM}-1}$ ) and 090055 (harboring $\left.b l a_{\mathrm{NDM}-5}\right)$, belong to 2 clones (38 SNPs between each other) which differed from the aforementioned clone by 81-82 SNPs. The relatively low number of SNPs among the 3 ST171 clones also suggests recent divergence within the lineage. In addition, the 6 ST171 strains in this study were clustered together with $2 b l a_{\mathrm{NDM}-1}-$ harboring strains, strain CCBH10892 isolated from Brazil in 2012, and strain EC_849 from South Africa in 2012, with 98 to 107 SNPs, but had >300 SNPs with other ST171 strains that had genome sequences deposited in GenBank. This finding suggests that the 6 strains identified in this study, CCBH10892, and EC_849 represent a subclade of ST171, which carries $b l a_{\mathrm{NDM}^{\prime}}$ has an international distribution, and might have emerged within the past 10 years. In addition, although 090055 and the other 4 bla ${ }_{\mathrm{NDM}-5}$-harboring strains (090011, 090022, 090023, and 090059) belonged to 2 different clones, they had the same IncX3 plasmid carrying $b l a_{\mathrm{NDM}-5^{\prime}}$ which suggests that the spread of $b l a_{\mathrm{NDM}-5}$ in the hospital was both clonal (vertical) and plasmidborne (horizontal).

The association of ST171 E. xiangfangensis with outbreaks is not rare; repeated reports from different geographic locations have demonstrated the same association (20-24). This association suggests that ST171 is a lineage of Enterobacter, which might be well adapted to causing infections in healthcare settings. Previous reports have demonstrated that ST171 E. xiangfangensis is a high-risk clone mediating the spread of carbapenem resistance $(21,23)$. Its emergence was initially documented in 2015 and 2016 by 2 studies in the United States $(24,25)$. Subsequent 
studies have revealed the international distribution of ST171 (21-23). Almost all carbapenem-resistant ST171 E. xiangfangensis strains have bla ${ }_{\mathrm{KPC}}(21)$, and only a small number of strains carry bla ${ }_{\mathrm{NDM}}$ instead $(21,26)$. In this study, we identified in-hospital transmission of carbapenem-resistant ST171 strains, which carried $b l a_{\mathrm{NDM}-5}$ rather than $b l a_{\mathrm{KPC}}$ as seen in previous studies $(21,23)$. The ability to acquire different carbapenemase genes and its adaptability to healthcare settings might be major drivers in the emergence of ST171, which warrants further study.

Our investigation demonstrates the value of whole-genome sequencing for precise species identification. However, this study has several limitations. First, because it is a single site study, the application of our findings could be limited. However, we analyzed genomes available in GenBank to provide the most comprehensive information possible. Second, the relatively small sample size in this study might not have adequate power to examine statistical significance in BSI type and patient outcomes. However, this study provided useful information on the clinical importance of E. xiangfangensis and its closely related taxon 1. Larger-scale studies are warranted.

In conclusion, most Enterobacter strains recovered from human blood in China were not E. cloacae but E. xiangfangensis. Most Enterobacter BSI cases in our study were healthcare-associated and primary infections. E. xiangfangensis ST171 is a major lineage of carbapenem-resistant Enterobacter, has an intercontinental distribution, is usually healthcare associated, and carries $b l a_{\mathrm{NDM}}$ rather than $b l a_{\mathrm{KPC}}$ in China. Precise species identification of Enterobacter has clinical importance in antimicrobial therapy and infection control.

Addendum: Since submission and acceptance of this manuscript, the taxonomy of Enterobacter has been substantially updated. The updated Enterobacter taxonomy is available at https://doi.org/10.1128/ mSystems.00527-20. The update of new taxa identified in this study is shown in Appendix 3 Table (https:/ / wwwnc.cdc.gov/EID/article/27/1/190154-App3.pdf).

\section{Acknowledgments}

We are grateful for Alan McNally for his proofreading and helpful suggestions.

The work was supported by grants from the National Natural Science Foundation of China (project no. 81772233, 81661130159 and 81861138055), West China Hospital of Sichuan University (1.3.5 project for disciplines of excellence, project no. ZYYC08006) and the Newton Advanced Fellowship, Royal Society, UK (NA150363).

\section{About the Author}

Dr. Wu is a scientist in the Department of Laboratory Medicine, West China Second Hospital, Sichuan University. Her major research interests are the epidemiology and clonal background of carbapenemaseproducing Enterobacter spp.

\section{References}

1. Mezzatesta ML, Gona F, Stefani S. Enterobacter cloacae complex: clinical impact and emerging antibiotic resistance. Future Microbiol. 2012;7:887-902. https://doi.org/10.2217/ fmb.12.61

2. Tindall BJ, Sutton G, Garrity GM. Enterobacter aerogenes Hormaeche and Edwards 1960 (approved lists 1980) and Klebsiella mobilis Bascomb et al. 1971 (approved lists 1980) share the same nomenclatural type (ATCC 13048) on the approved lists and are homotypic synonyms, with consequences for the name Klebsiella mobilis Bascomb et al. 1971 (approved lists 1980). Int J Syst Evol Microbiol. 2017;67:502-4. https://doi.org/10.1099/ijsem.0.001572

3. Gavini R, Mergaert J, Beji A, Mielcarek C, Izard D, Kersters K, et al. Transfer of Enterobacter agglomerans (Beijerinck 1888) Ewing and Fife 1972 to Pantoea gen. nov. as Pantoea agglomerans comb. nov. and description of Pantoea dispersa sp. nov. Int J Syst Evol Microbiol. 1989;39:337-45.

4. Iversen C, Mullane N, McCardell B, Tall BD, Lehner A, Fanning $S$, et al. Cronobacter gen. nov., a new genus to accommodate the biogroups of Enterobacter sakazakii, and proposal of Cronobacter sakazakii gen. nov., comb. nov., Cronobacter malonaticus sp. nov., Cronobacter turicensis sp. nov., Cronobacter muytjensii sp. nov., Cronobacter dublinensis sp. nov., Cronobacter genomospecies 1 , and of three subspecies, Cronobacter dublinensis subsp. dublinensis subsp. nov., Cronobacter dublinensis subsp. lausannensis subsp. nov. and Cronobacter dublinensis subsp. lactaridi subsp. nov. Int J Syst Evol Microbiol. 2008;58:1442-7. https://doi.org/10.1099/ijs.0.65577-0

5. Hoffmann H, Roggenkamp A. Population genetics of the nomenspecies Enterobacter cloacae. Appl Environ Microbiol. 2003;69:5306-18. https:/ / doi.org/10.1128/AEM.69.9. 5306-5318.2003

6. Hoffmann H, Stindl S, Stumpf A, Mehlen A, Monget D, Heesemann J, et al. Description of Enterobacter ludwigii sp. nov., a novel Enterobacter species of clinical relevance. Syst Appl Microbiol. 2005;28:206-12. https:/ / doi.org/10.1016/ j.syapm.2004.12.009

7. Wu W, Feng Y, Zong Z. Enterobacter sichuanensis sp. nov., recovered from human urine. Int J Syst Evol Microbiol. 2018;68:3922-7. https://doi.org/10.1099/ijsem.0.003089

8. Balloux F, Brønstad Brynildsrud O, van Dorp L, Shaw LP, Chen H, Harris KA, et al. From theory to practice: translating whole-genome sequencing (WGS) into the clinic. Trends Microbiol. 2018;26:1035-48. https:/ / doi.org/ 10.1016/j.tim.2018.08.004

9. Besser J, Carleton HA, Gerner-Smidt P, Lindsey RL, Trees E. Next-generation sequencing technologies and their application to the study and control of bacterial infections. Clin Microbiol Infect. 2018;24:335-41. https://doi.org/ 10.1016/j.cmi.2017.10.013

10. Rosselló-Móra R, Amann R. Past and future species definitions for Bacteria and Archaea. Syst Appl Microbiol. 2015;38:209-16. https://doi.org/10.1016/j.syapm. 2015.02.001 
11. Clinical and Laboratory Standards Institute. Performance standards for antimicrobial susceptibility testing; twentyseventh informational supplement. M100-S27. Wayne (PA): The Institute; 2017.

12. Magiorakos AP, Srinivasan A, Carey RB, Carmeli Y, Falagas ME, Giske CG, et al. Multidrug-resistant, extensively drug-resistant and pandrug-resistant bacteria: an international expert proposal for interim standard definitions for acquired resistance. Clin Microbiol Infect. 2012;18:268-81. https://doi.org/10.1111/j.1469-0691.2011.03570.x

13. Centers for Disease Control and Prevention. National Healthcare Safety Network FAQs: bloodstream infection (BSI) events. 2018 [cited 2018 Nov 1]. https://www.cdc.gov/ nhsn/faqs/faq-bsi.html

14. Centers for Disease Control and Prevention. Identifying healthcare-associated infections (HAI) for NHSN surveillance. 2018 [cited 2018 Nov 1]. https:/ / www.cdc.gov/ nhsn/pdfs/pscmanual/2psc_identifyinghais_nhsncurrent.pdf

15. Wick RR, Judd LM, Gorrie CL, Holt KE. Unicycler: resolving bacterial genome assemblies from short and long sequencing reads. PLOS Comput Biol. 2017;13:e1005595. https://doi.org/10.1371/journal.pcbi.1005595

16. Meier-Kolthoff JP, Auch AF, Klenk HP, Göker M. Genome sequence-based species delimitation with confidence intervals and improved distance functions. BMC Bioinformatics. 2013;14:60. https://doi.org/10.1186/ 1471-2105-14-60

17. Stamatakis A. RAxML version 8: a tool for phylogenetic analysis and post-analysis of large phylogenies. Bioinformatics. 2014;30:1312-3. https:/ / doi.org/10.1093/bioinformatics/ btu033

18. Walker BJ, Abeel T, Shea T, Priest M, Abouelliel A, Sakthikumar S, et al. Pilon: an integrated tool for comprehensive microbial variant detection and genome assembly improvement. PLoS One. 2014;9:e112963. https:/ / doi.org/10.1371/journal.pone.0112963

19. Pati NB, Doijad SP, Schultze T, Mannala GK, Yao Y, Jaiswal S, et al. Enterobacter bugandensis: a novel enterobacterial species associated with severe clinical infection. Sci Rep. 2018;8:5392. https:/ / doi.org/10.1038/ s41598-018-23069-z
20. Hawken SE, Washer LL, Williams CL, Newton DW, Snitkin ES. Genomic investigation of a putative endoscopeassociated carbapenem-resistant Enterobacter cloacae outbreak reveals a wide diversity of circulating strains and resistance mutations. Clin Infect Dis. 2018;66:460-3. https://doi.org/ 10.1093/cid/cix934

21. Chavda KD, Chen L, Fouts DE, Sutton G, Brinkac L, Jenkins SG, et al. Comprehensive genome analysis of carbapenemaseproducing Enterobacter spp.: new insights into phylogeny, population structure, and resistance mechanisms. MBio. 2016;7:02093-16. https:// doi.org/10.1128/mBio.02093-16

22. Harada K, Shimizu T, Mukai Y, Kuwajima K, Sato T, Kajino A, et al. Phenotypic and molecular characterization of antimicrobial resistance in Enterobacter spp. isolates from companion animals in Japan. PLoS One. 2017;12:e174178. https://doi.org/10.1371/journal.pone.0174178

23. Gomez-Simmonds A, Annavajhala MK, Wang Z, Macesic N, $\mathrm{Hu}$ Y, Giddins MJ, et al. Genomic and geographic context for the evolution of high-risk carbapenem-resistant Enterobacter cloacae complex clones ST171 and ST78. MBio. 2018;9:0054218. https://doi.org/10.1128/mBio.00542-18

24. Hargreaves ML, Shaw KM, Dobbins G, Snippes Vagnone PM, Harper JE, Boxrud D, et al. Clonal dissemination of Enterobacter cloacae harboring $b l a_{\mathrm{KPC}-3}$ in the upper midwestern United States. Antimicrob Agents Chemother. 2015;59:772334. https://doi.org/10.1128/AAC.01291-15

25. Gomez-Simmonds A, Hu Y, Sullivan SB, Wang Z, Whittier S, Uhlemann AC. Evidence from a New York City hospital of rising incidence of genetically diverse carbapenem-resistant Enterobacter cloacae and dominance of ST171, 2007-14. J Antimicrob Chemother. 2016;71:2351-3. https://doi.org/10.1093/jac/dkw132

26. Peirano G, Matsumura Y, Adams MD, Bradford P, Motyl M, Chen L, et al. Genomic epidemiology of global carbapenemaseproducing Enterobacter spp., 2008-2014. Emerg Infect Dis. 2018;24:1010-9. https://doi.org/10.3201/eid2406.171648

Address for correspondence: Zhiyong Zong, Center of Infectious Diseases, West China Hospital (Huaxi), Guoxuexiang 37, Chengdu 610041, China; email: zongzhiy@scu.edu.cn 